\title{
Improving Phase Contrast of Digital Holographic Microscope using Spatial Light Modulator
}

\author{
Le Thanh Bang ${ }^{1}$, Meilan Piao ${ }^{1}$, Jong-Rae Jeong ${ }^{2}$, Seok-Hee Jeon ${ }^{3}$, and Nam Kim ${ }^{1 *}$ \\ ${ }^{l}$ School of Electrical Engineering and Computer Science, Chungbuk National University, \\ 1 Chungdae-ro, Seowon-gu, Cheongju 362-763, Korea \\ ${ }^{2}$ Department of Information and Communication, Suwon Science College, \\ 288 Seja-ro, Jeongnam-myeon, Hwaseong 445-742, Korea \\ ${ }^{3}$ Department of Electronics Engineering, Incheon National University, 119 Academy-ro, Yeonsu-gu, \\ Incheon 406-772, Korea
}

(Received August 5, 2014 : revised October 13, 2014 : accepted October 17, 2014)

\begin{abstract}
We propose a new method for improving the phase contrast of a multiphase digital holographic microscope using a spatial light modulator (SLM). Using the SLM as the annulus, our method improves the light contrast of the object edge to achieve higher accuracy. We demonstrate a digital holographic microscopy technique that provides a $30 \%$ improvement in the phase contrast compared to conventional microscopy, which utilizes a mechanical annulus. The phase-contrast improvement allows the 3D reconstructed hologram to be determined more precisely.
\end{abstract}

Keywords : Digital holography, Microscopy, Phase-shifting interferometry

OCIS codes : (090.0090) Holography; (090.2880) Holographic interferometry; (170.0180) Microscopy;

(170.2520) Fluorescence microscopy

\section{INTRODUCTION}

Contrast microscopy is very useful for observing specimens and studying biological processes because of its ability to examine cells in their natural state. Observing a living organism in its natural state or environment can provide more information than observing specimens that need to be killed, fixed, or stained before microscopy. Reconstructed three-dimensional (3D) living cells have been studied in contrast microcopy with high-contrast [1-3], high-resolution images [3]. When combined with other means of observation such as fluorescence [4-5], contrast microscopy can be used for studying and interpreting thin specimens [5]. In addition, advances in phase-contrast microscopy, enable us to concentrate on the minute internal structures of particles [6] and can detect a very small number of protein molecules [7].

The in-line digital holographic microscope (DHM) [8-10] is a promising system that gives good performance in 3D reconstruction measurements of specimens. The DHM provides quantitative phase contrast imaging suitable for high-resolution investigations on reflective surfaces as well as for marker- free analysis of living cells [11, 12]. Furthermore, the focus adjustment can be carried out numerically after the measurement, and mechanical auto focus tracking during the measurement is avoided. Thus, DHM offers the advantage of multiple focus plane reconstruction from a single hologram [12]. However, when a thick specimen is used as an object, the images are often surrounded by bright areas, which obscure details along the perimeter of the specimen. These bright regions occur because of the fixed phase of a specially designed annular diaphragm, which is equal in diameter and optically conjugate to an internal phase plate residing in the objective rear focal plane. Depending on the position of the living cell, the phase of light may be one-half or one-quarter wavelength out of phase. As a result, the accuracy of the reconstructed 3D cell is decreased. On other the hand, the phase plate and phase annulus in phase contrast microscopy use special materials as absorbing or dielectric film [13], and the phase difference is calculated based on the thickness and refractive index of these materials [14]. Therefore, mechanical adjustments are required to calculate the distance between the objective lens and the condenser

\footnotetext{
*Corresponding author: namkim@chungbuk.ac.kr

Color versions of one or more of the figures in this paper are available online.
} 
lens with the specimen plate.

In this study, we demonstrate a new method for obtaining phase shifting with a higher phase contrast in the reconstructed 3D hologram using a spatial light modulator (SLM) and an in-line DHM [16]. The key component of this method is an SLM that can display phase information. Using this SLM, the phase band rings can be easily modified with computer software for different sizes and radii. By changing the phase band rings, this method can record phase shifting in a reconstructed specimen that has different thicknesses, removing the dependence on the fixed phase of the phase plate and phase annulus used in conventional phase contrast microscopy. Therefore, the proposed system allows flexibility in the phase choice by calculating the phase on the annulus plane in accordance with the thickness of each object in order to observe the object more precisely.

After describing our proposed method in Section 2, two experimental setups are described in detail in Section 3. The first setup is an optical experiment demonstrating the direct observation of pollen while changing the phase band rings on SLM. The second experiment demonstrates a recording hologram using the fluorescent dye specimen B10F16. Finally, the reconstructed image of the specimen can be seen more clearly than conventional phase contrast microscopy with a contrast improvement of about $30 \%$.

\section{PROPOSED METHOD}

By following the optical principle of phase contrast microscopy as well as the principle of holography, we assume that the laser beam with wavelength $\lambda$ output from the laser source is collimated, creating a plane wave that propagates to the specimen plate. The plane wave is expressed as

$$
f(\boldsymbol{X})=A e^{i \varphi}
$$

where $X=\left\{\left(x_{j}^{r}, x_{j}^{c}\right), j=1, \ldots, N\right\}$ describes the row and column (2D locations) of the total number of image pixels $\mathrm{N}$ on the specimen plate, and $\mathrm{A}$ and $\varphi$ are the amplitude and phase of the illuminating wave before entering the specimen plate, respectively.

After the illuminating wave passes through the specimen plate, the wave is divided two components: the unaltered surround wave $f(X)$ and the diffracted wave $g(X)$ that is attenuated and had its phase changed by the specimen. The functions of the two waves are described as

$$
\begin{aligned}
& f(\boldsymbol{X})=A e^{i \varphi} \\
& g(\boldsymbol{X})=\Upsilon_{c} e^{-i O(x)} f(\boldsymbol{X})=\Upsilon_{C} A e^{i(\varphi-O(x))}
\end{aligned}
$$

where $\gamma_{c}$ is the amplitude attenuation, and $O(X)$ is the phase shift created by the specimen at location $x$.

Due to the illuminating waves $f(X)$ and $g(X)$ passing

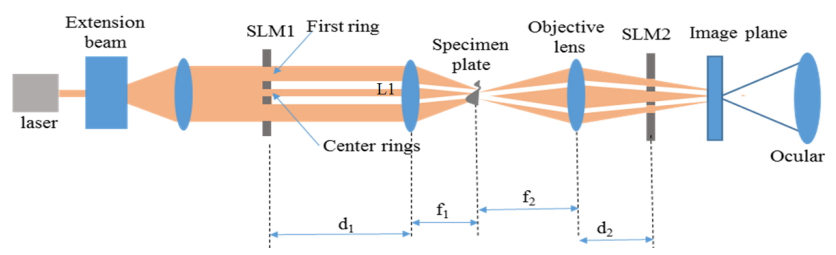

FIG. 1. Proposed structure of the basic contrast phase microscope using SLMs with a 60x objective lens.

through the thin lens $\mathrm{L}_{1}$, as shown in Fig. 1, we can express the Fourier transform of this wave as follows:

$$
F(w)=\mathcal{F}(f(\boldsymbol{X})) \text { and } G(w)=\mathcal{F}(g(\boldsymbol{X}))
$$

We assume that the phase plate is an SLM that includes a band ring. This band ring is similar to a band-pass filter, and the unaltered surround wave $f(X)$ advances its phase by quarter-wavelength $\pi / 2$. Thus, the corresponding transmittance function for the surround wave is

$$
U_{f}(w)=\Upsilon_{A} e^{\pi / 2}=i \Upsilon_{A}
$$

where $\gamma_{A}$ represents the amplitude attenuation by the phase ring on SLM with radius $\mathrm{R}$ and width $\mathrm{W}$. The diffracted wave on the phase plate leaks a small portion of the wave into the ring. The ring acts like a band-pass filter as follows:

$$
U_{g}(w)=1+\left(i \Upsilon_{A}-1\right)\left[\operatorname{cyl}\left(\frac{w_{r}}{R}\right)-c y l\left(\frac{w_{r}}{R-W}\right)\right]
$$

where $W_{r}=\sqrt{W_{u}^{2} W_{v}^{2}}$ is the radial frequency, and $c y l$ is a 2D cylinder function display on SLM, expressed by

$$
\operatorname{cyl}(w)=\left\{\begin{array}{c}
1 \quad \text { if } 0 \leq w \leq 1 \\
0 \text { otherwise }
\end{array}\right.
$$

After the SLM, we have the following waves:

$$
\begin{aligned}
& \tilde{F}(w)=F(w) U_{f}(w) \\
& \tilde{G}(w)=G(w) U_{g}(w)
\end{aligned}
$$

The ocular lens performs another Fourier transform, as follows:

$$
\begin{aligned}
& \tilde{f}(X)=f(X) * u_{f}(X) \\
& \tilde{g}(X)=g(X) * u_{g}(X)
\end{aligned}
$$

where $u_{f}(X)=i \gamma_{A} \delta(X)$, and $u_{g}(X)=\delta(r)+\left(i \gamma_{A}-1\right)$ airy (R),

Thus, the intensity of the final observed image [15] is calculated by 


$$
g(X)=(\delta(\mathrm{r})-\operatorname{airy}(r)) * O(X)+C
$$

This function represents the point-spread function of phase contrast microscope:

$$
\operatorname{PSF}(u, v)=\delta(u, v)-\operatorname{airy}\left(\sqrt{u^{2}+v^{2}}\right)
$$

Similarly, in the annulus SLM position, we have an even number of phases with $n$ band rings, which have opposite phases. For example, the first band ring has phase $\varphi$, the second band ring has phase $-\varphi$, and so on. Then, the function of the phase contrast microscope is

$$
\operatorname{PSF}(u, v)=\frac{n \varphi}{\pi}\left[\delta(u, v)-\operatorname{airy}\left(\sqrt{u^{2}+v^{2}}\right)\right]
$$

where $\varphi$ satisfies $0 \leq \varphi \leq \frac{\pi}{2}$, and $n$ is number of band rings on SLM.

So, we can calculate a hologram from point-spread function of phase contrast microscope by function:

$$
U_{h}(x, y)=e^{j \frac{\pi}{\lambda z}\left(x^{2}+y^{2}\right)} \int_{-\infty}^{\infty} P S F(u, v) e^{j \frac{\pi}{\lambda z}\left(u^{2}+v^{2}\right)} \exp \left[-j \frac{\pi}{\lambda z}\left(u^{2}+v^{2}\right)\right] d u d v
$$

From Eq. (13) to increase the contrast between the pixels, the phase of the band rings on SLM1 have to satisfy the condition $\frac{n \varphi}{\pi} \geq 1$ or $\varphi \geq \frac{\pi}{n}$. In other words, on an SLM with four band rings of opposing phase, then the phase of each band ring is larger than $\frac{\pi}{4}$.

The relative size of the phase band rings between SLM1 and SLM2 can be described as follows:

$$
W_{S L M 1}=\frac{f_{2}\left(d_{1}-f_{1}\right)}{f_{1}} W_{S L M 2}
$$

where $W_{S L M}$ and $W_{S L M 2}$ is the width of band rings on SLM1 and SLM2, respectively, $d_{1}$ is the distance from SLM1 to lens 1 , and $f_{1}$ and $f_{2}$ are the focal lengths of lens 1 and the objective lens, respectively.

Finally, the distance from the objective lens to SLM2 is

$$
d_{2}=f_{1}+f_{2}-\frac{f_{1} d_{1}}{d_{1}-f_{1}}
$$

\section{EXPERIMENT}

In the first experiment, we captured the images of an object using the optical setup shown in Fig. 1. We applied the proposed method, changing the number of $f$ band rings on SLM1 and SLM2. The laser beam with a 532-nm wavelength and output power of $150 \mathrm{~mW}$ is expanded, collimated, and passed through SLM1, which is placed $30 \mathrm{~mm}$ from lens $L_{1}$ with a $100-\mathrm{mm}$ focal length. Here, $\mathrm{L}_{2}$ is a $60 \mathrm{x}$ magnifying microscopic objective. The object is the pollen from a rhododendron placed on a thin glass plate. The CCD camera has $1024 \times 768$ pixels, with each pixel having an area of $9 \times 9 \mu \mathrm{m}^{2}$ and 8-bit resolution.

Figure 3 shows contrast phase microscopy with different band rings on SLM1 and SLM2. From Fig. 3(a) to 3(d), the number of band rings is increased from two band rings with phase 0 and $\frac{\pi}{2}$, four band rings with phase $0, \frac{\pi}{2}$; $\frac{\pi}{4}$ and $\frac{7 \pi}{4}$ six band rings with phase $0, \frac{\pi}{2} ; \frac{\pi}{4}, \frac{7 \pi}{4}$; $\frac{3 \pi}{8}$ and $\frac{13 \pi}{8}$, and eight band rings with phase $0, \frac{\pi}{2}, \frac{\pi}{4}$, $\frac{7 \pi}{4}, \frac{3 \pi}{8}, \frac{13 \pi}{8}, \frac{\pi}{8}$ and $\frac{15 \pi}{8}$. In Fig. 3(e) and 3(f), the order of phase couples is changed from Fig. 3(d). In particular, in Fig. 3(e), the two band rings with phases 0 , $\frac{\pi}{2}$ are located on the outermost band rings, while the band rings with phase $\frac{\pi}{4}, \frac{7 \pi}{4}, \frac{3 \pi}{8}, \frac{13 \pi}{8}, \frac{\pi}{8}, \frac{15 \pi}{8}$ are located from the inside to the outside; whereas in Fig. 3(f), the two outermost band rings have phases $\frac{\pi}{4}, \frac{7 \pi}{4}$ with band rings of phase $\frac{3 \pi}{8}, \frac{13 \pi}{8}, \frac{\pi}{8}, \frac{15 \pi}{8}, 0, \frac{\pi}{2}$ located from the inside to the outside. These images show a significant increase in contrast of the interference edge of the pollen when band rings with multiple phases are utilized. In the experiment, a standard environmental plane wave was created when the light source passed through a spatial filter and lens. The interfered light on the CCD image sensor was adjusted to an average intensity of the mono mode, which was maintained for all six cases.

Figure 4 describes the pixel value of the CCD camera on the $420^{\text {th }}$ line in the images. This figure show the data

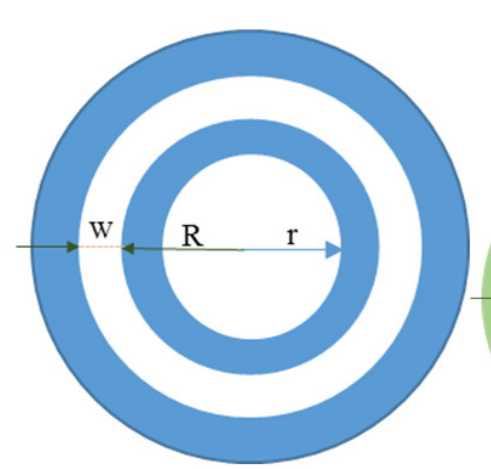

(a)

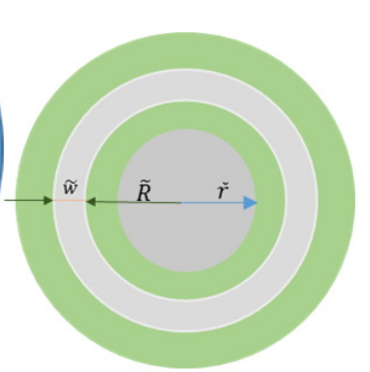

(b)
FIG. 2. The relative size of the phase band rings between (a) SLM1 and (b) SLM2. 
of the recorded images with a phase contrast of two band rings (Fig. 3(a)), four band rings (Fig. 3(b)), six band rings (Fig. 3(c)) and eight band rings (Fig. 3(d)) on SLM1 and SLM2, respectively. The level pixel value of each edge on the pollen is described by the red line in this figure. In Fig. 4, the pixel value is "blue dot" and the solid

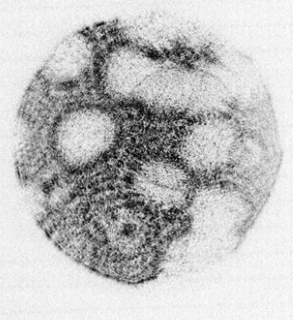

(a)

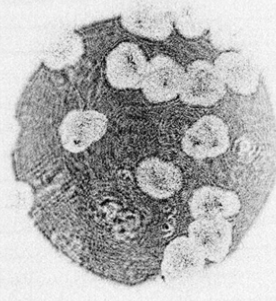

(d)

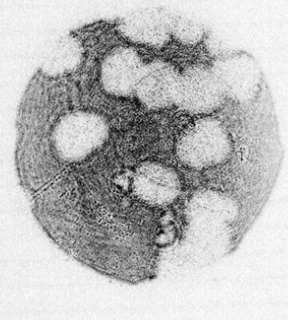

(b)

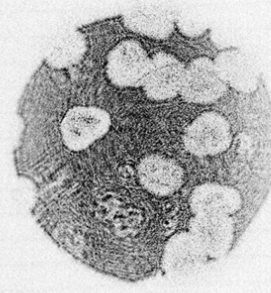

(e)

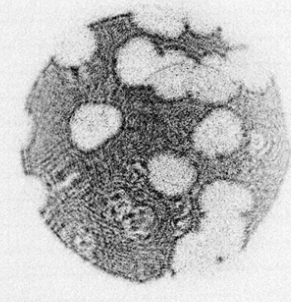

(c)

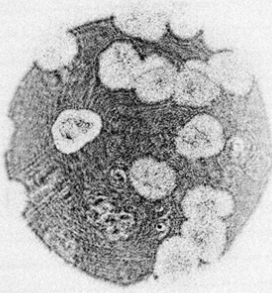

(f)

FIG. 3. Recording images contrast phase (a) two band rings, (b) four band rings, (c) six band rings, (d) eight band rings with phase $0, \frac{\pi}{2} ; \frac{\pi}{4}, \frac{7 \pi}{4} ; \frac{3 \pi}{8}, \frac{13 \pi}{8} ; \frac{\pi}{8}$ and $\frac{15 \pi}{8}$, (e) eight band rings with phase: $\frac{\pi}{4}, \frac{7 \pi}{4} ; \frac{3 \pi}{8}, \frac{13 \pi}{8} ; \frac{\pi}{8}, \frac{15 \pi}{8} ; 0$ and $\frac{\pi}{2}$ (f) eight band rings with phase: $\frac{3 \pi}{8}, \frac{13 \pi}{8} ; \frac{\pi}{8}, \frac{15 \pi}{8} ; 0, \frac{\pi}{2} ; \frac{\pi}{4}$ and $\frac{7 \pi}{4}$.

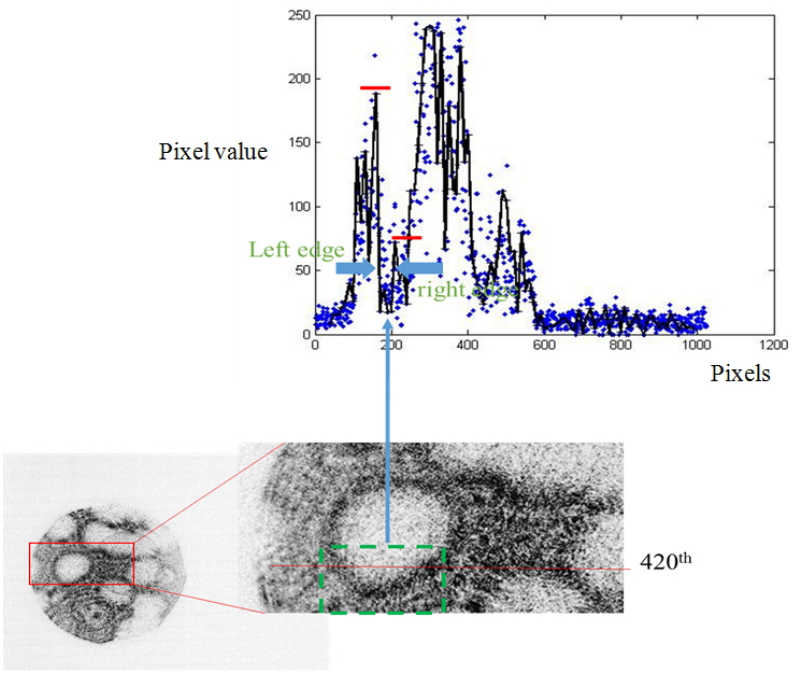

(a)

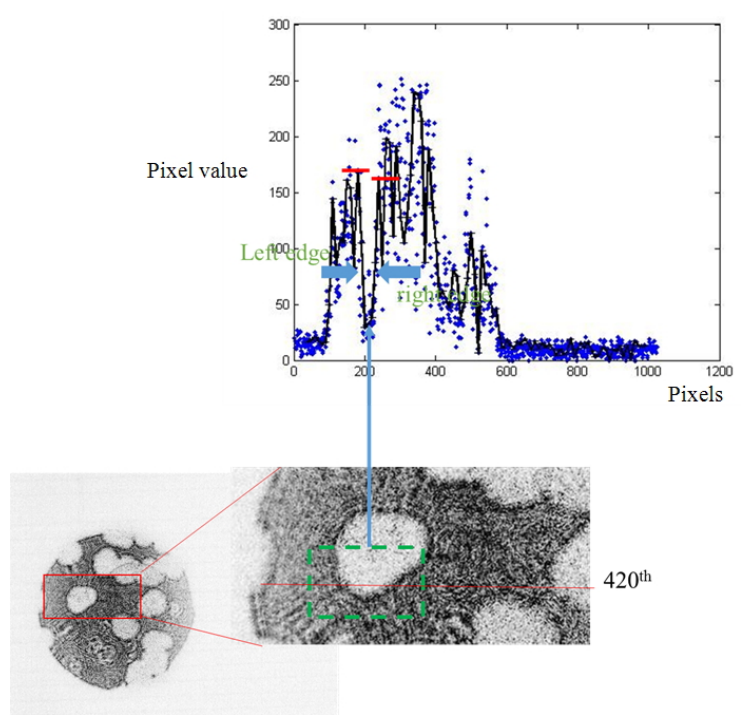

(b)

FIG. 4. (a) The pixel values on the cross-section in line $420^{\text {th }}$ for (a) two band ring, (b) four band rings, (c) six band rings and (d) eight band rings with phase (Continued). 


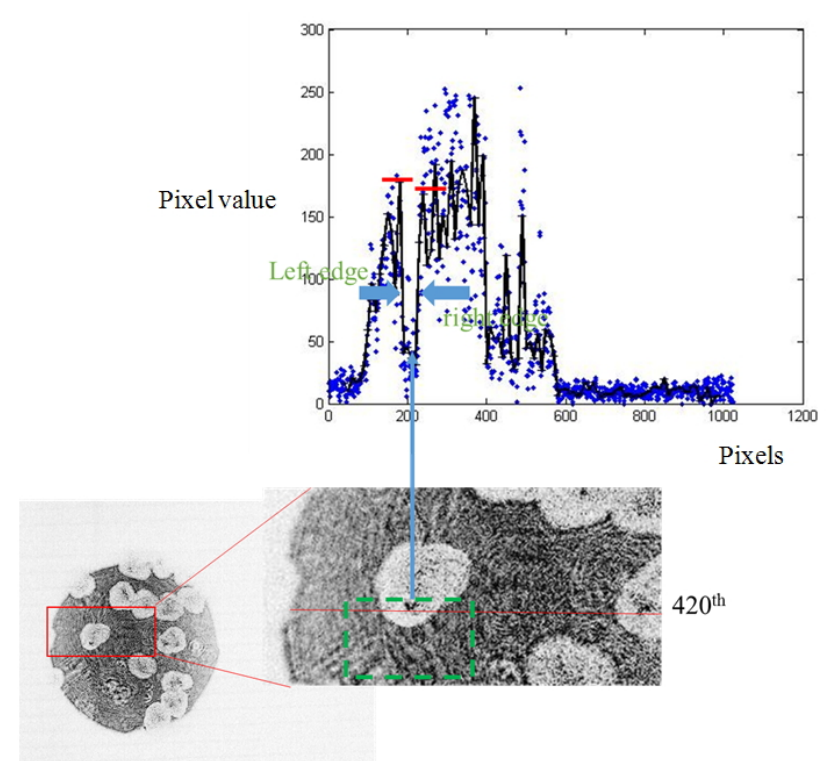

(c)

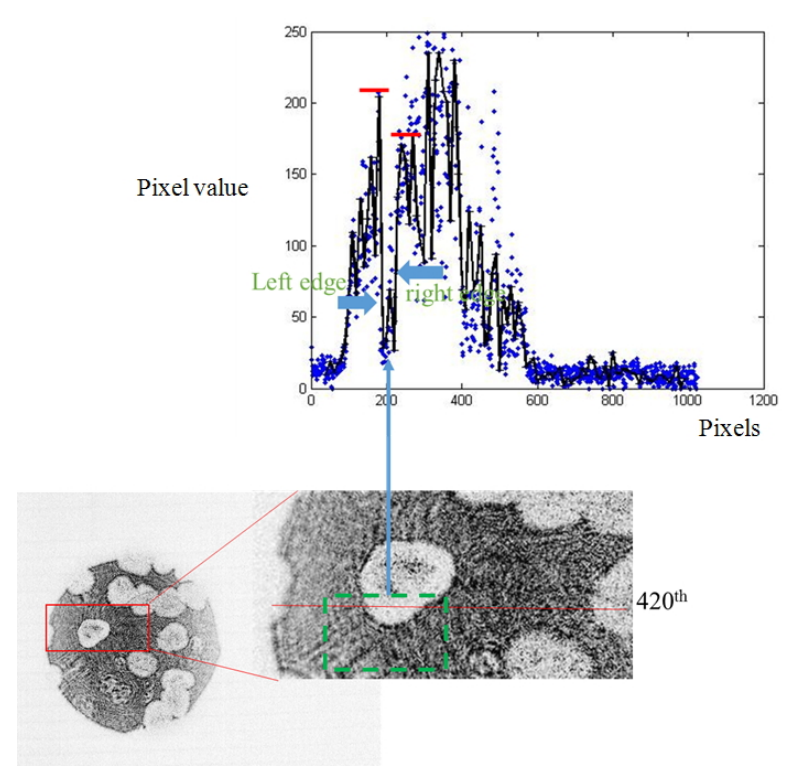

(d)

FIG. 4. (a) The pixel values on the cross-section in line $420^{\text {th }}$ for (a) two band ring, (b) four band rings, (c) six band rings and (d) eight band rings with phase.

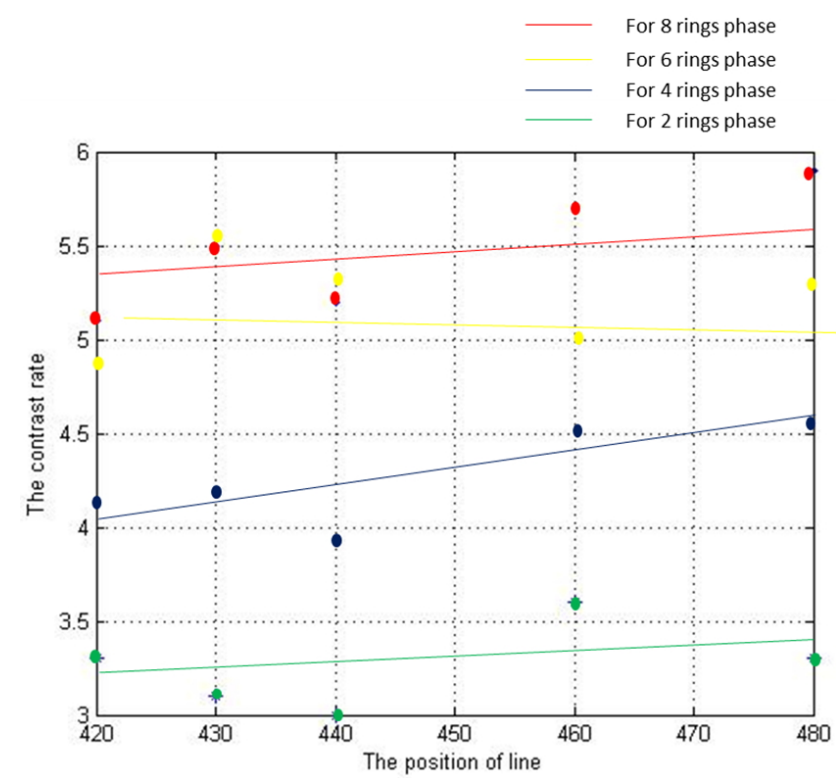

FIG. 5. The contrast rate of the pixel values at the pollen edges.

line is the average of each ten pixels on this cross line.

The proposed method with eight band rings has a higher contrast than that with two band rings. The edge of the pollen at the $420^{\text {th }}$ line with eight band rings on the SLM is about $39.3 \mu \mathrm{m}$, as shown in Fig. 4(d), while that with two band rings is greater than $50 \mu \mathrm{m}$, as shown in Fig. 4(a). The pixel value on the $420^{\text {th }}$ on the left side began to change from $180^{\text {th }}$ to $185^{\text {th }}$. Therefore, to evaluate the contrast between the edge of pollen and the outside environment, we calculated the ratio of the average value of the

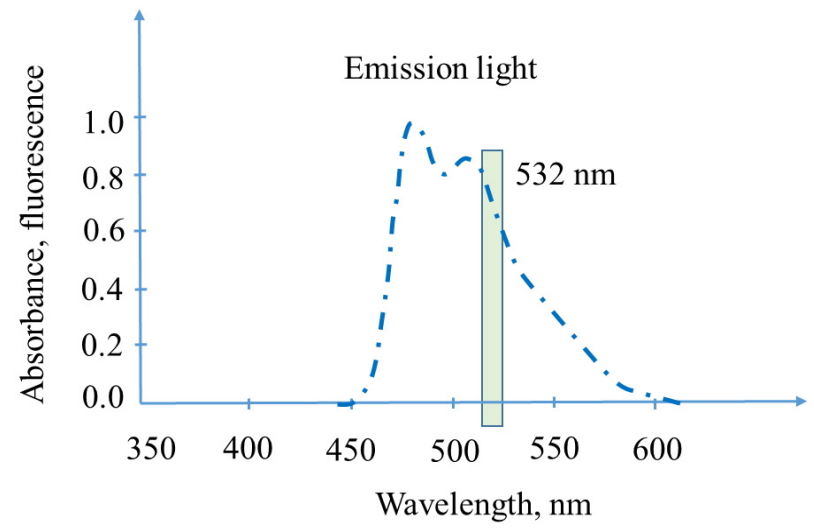

FIG. 6. The emission wavelengths of green fluorescent protein (GFP).

10 pixels from $175^{\text {th }}$ to $185^{\text {th }}$ and 10 pixels from $185^{\text {th }}$ to $195^{\text {th }}$. In Fig. 4(a), the average of 10 pixel value from $175^{\text {th }}$ to $185^{\text {th }}$ is 142 and 10 pixels from $185^{\text {th }}$ to $195^{\text {th }}$ is 43 so the rate is approximately $142 / 43 \approx 3.3$, while in Fig. $4(d)$, this rate is about $185 / 36 \approx 5.13$. This contrast rate is described in Fig. 5 with different line for $430^{\text {th }}, 440^{\text {th }}$, $460^{\text {th }}$ and $480^{\text {th }}$.

In the second experiment, we captured an in-line hologram of a living cell that was dyed with green fluorescent protein (GFP). The emission spectra wavelengths of this GFP are approximately 450nm to $600 \mathrm{~nm}$ (Fig. 6), amplitude emission peak is at $470 \mathrm{~nm}$ and declines with increasing wavelength [17]. The wavelength $532 \mathrm{~nm}$ is chosen in this experiment for recording the hologram.

The optical setup is illustrated in Fig. 7. A femtosecond laser with 900-nm wavelength is used as the exciting 


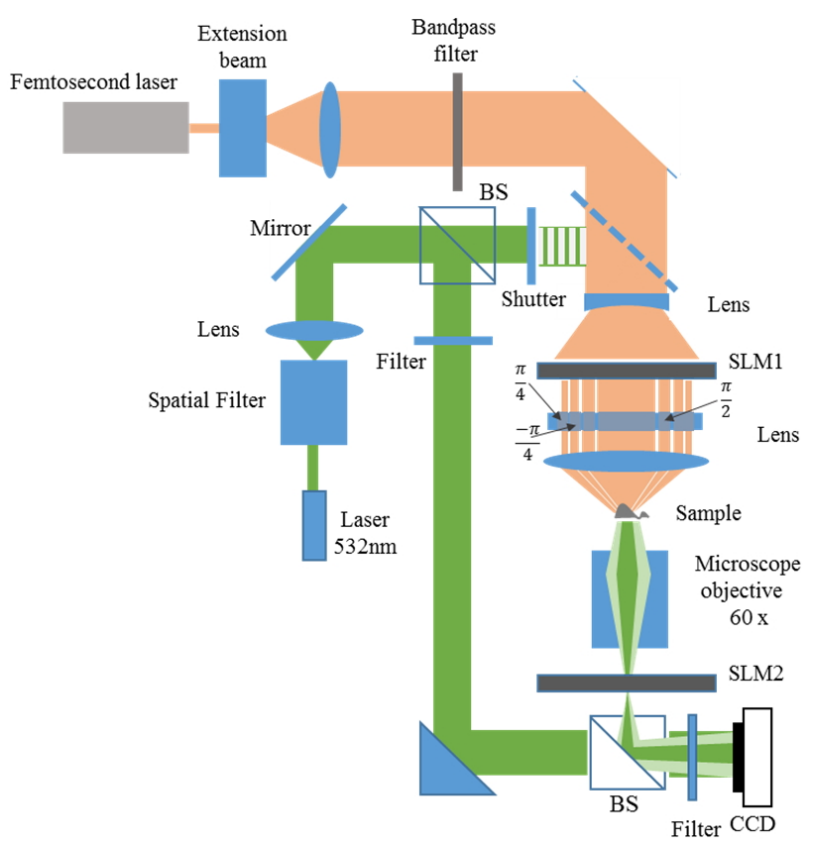

FIG. 7. Experimental setup for recording a hologram using the proposed method.

beam, and the experiment is performed similarly to the principles outlined in Fig. 1. A laser beam with a wavelength of $532 \mathrm{~nm}$ was used as a reference beam. This beam is separated into two beams: the first beam is a reference beam and recorded by $\mathrm{CCD}$ camera, the second beam is to check the quality hologram in the processing recording hologram. The second beam is turned off/ on by a shutter and it does not participate in the process of recording a hologram of the object cell.

In order to obtain the exact hologram of the specimen using the principle of holography, the light that has passed through the specimen must be filtered at a wavelength of $532 \mathrm{~nm}$ and must be calibrated carefully as follows. As the specimen was moved from the position of $\mathrm{z}=0.3 \mu \mathrm{m}$ to $2.6 \mu \mathrm{m}$ at intervals of $120 \mathrm{~nm}$, each hologram was recorded, and the axial position on the CCD plane was obtained from the diffraction calculation of the respective holograms, template matching, and focused objective matching.

Figure 8 shows the reconstructed hologram of specimen (B16F10) fixed on the substrate glass at the position of $\mathrm{z}=1.1 \mu \mathrm{m}$ from the focal plane of the objective lens. The images were captured with two phase band rings ( 0 and $\pi / 2$ ), six phase band rings $\left(0, \frac{\pi}{2}, \frac{\pi}{4}, \frac{7 \pi}{4}, \frac{3 \pi}{8}\right.$ and $\left.\frac{13 \pi}{8}\right)$, and eight phase band rings. These reconstructed images show significant increase in contrast as the number of phase band rings on the SLM increased from two to eight phase band rings. The improvements in contrast were realized by using the phase band rings on SLM instead of mechanical adjustment on the annulus, which increased the accuracy of the image of the specimen.

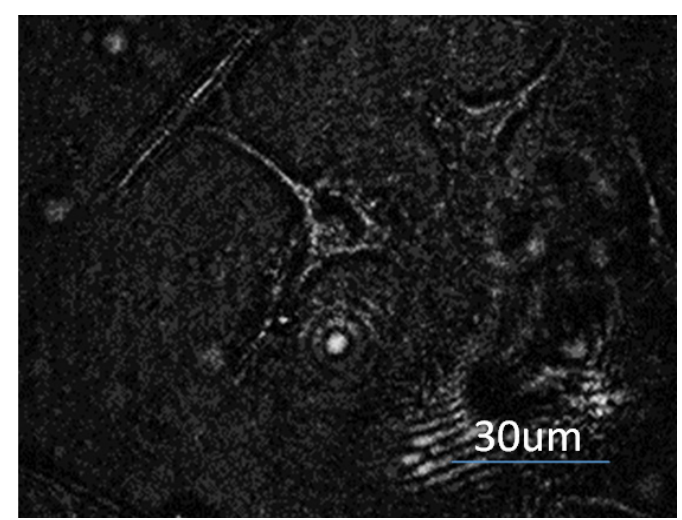

(a)

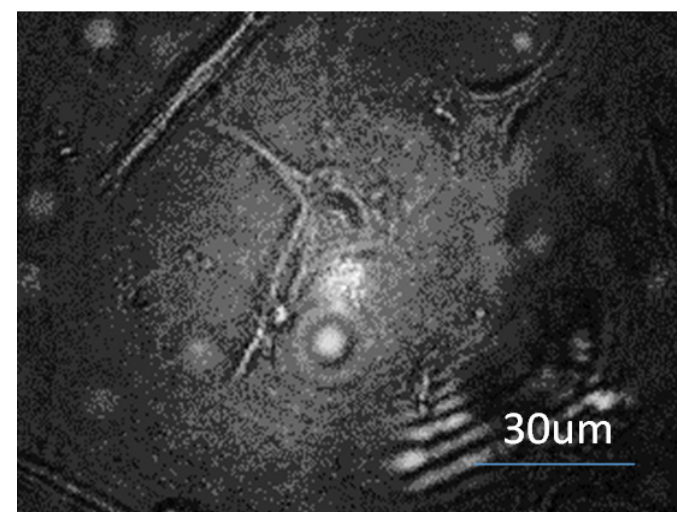

(b)

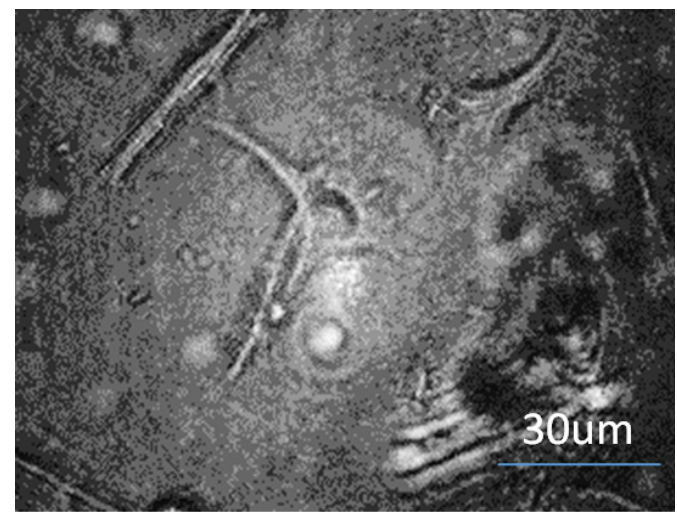

(c)

FIG. 8. Reconstructed images of the hologram specimen B16F10 with (a) two phase band rings, (b) six phase band rings, and (c) eight phase band rings (Media 1).

\section{CONCLUSION}

We developed a new type of annulus in digital holographic microscopy using two SLMs by changing the phase of the band rings. This method overcomes the limitations of conventional microscopy, which uses a mechanical annulus. We achieved flexibility in changing the phase and in choosing the number of phases to increase the contrast in images. When the object is reconstructed with holographic principles, our method improves the quality of the inter- 
ference edge of the micro-object (pollen) as well as the accuracy of the 3D living cell. Other advantages of our method include the ease of implementation and high stability of the optical system. However, the weakness of fluorescence holographic microscopy is that emission light comes only from the locations on the nucleus of cells or cell components that contain more protein, where dried fluorescence which has the same wavelength with the reference beam, so the cell wall is not clear. We believe that our proposed method, which involves the control of the phase contrast using SLM as well as the balance of the object and reference beam intensity in holographic microscopy, is a flexible and useful technique for advanced microscopy.

\section{ACKNOWLEDGMENT}

This work was supported by the National Research Foundation of Korea grant funded by the Korea Government (MSIP) (2014R1A2A2A01003934) and the Korea Creative Content Agency in the Culture Technology Research \& Development Program (R2013-08003).

\section{REFERENCES}

1. M. Kim, Y. Choi, W. Choi, and K. Kim, "Three-dimensional differential interference contrast microscopy using synthetic aperture imaging," J. Biomed Opt. 17, 26-30 (2012).

2. S. Chowdhury and J. Izatt, "Structured illumination quantitative phase microscopy for enhanced resolution amplitude and phase imaging," Biomed Opt. Express 10, 795-805 (2013).

3. X. Qu, C. Yang, and G. Zheng, "Quantitative phase imaging via Fourier ptychographic microscopy,” Opt. Lett. 38, 4845-4848 (2013).

4. A. Mark and W. David, "High-contrast imaging of fluorescent protein FRET by fluorescence polarization microscopy," Biophys. J. 88, 14-18 (2005).
5. T. Toney, P. Haro, B. Sotillo, and J. Solis, "Ion migration assisted inscription of high refractive index contrast waveguides by femtosecond laser pulses in phosphate glass," Opt. Lett. 38, 5248-5251 (2013).

6. G. Ritter, R. Veith, J. Siebrasse, and U. Kobitscheck, "High-contrast single-particle tracking by selective focal plane illumination microscopy," Opt. Express 16, 7142-7152 (2008).

7. J. Huisken, J. Swoger, F. Del Bene, J. Wittbrodt, and E. H. Stelzer, "Optical sectioning deep inside live embryos by selective plane illumination microscopy," Science 305, 10071009 (2004)

8. T. Shimobaba, H. Yamanashi, and I. Tomoyoshi, "In-line digital holographic microscopy using a consumer scanner," Nature 3, 1038-1048 (2013).

9. T. Vu, N. Kim, and C. Nam, "Simple phase-only optical decryption with misalignment-free input," Opt. Lett. 32, 223-225 (2007).

10. H. Cho, D. Kim, Y. Yu, W. Jung, and S. Shin, "Dualwavelength digital holography microscope for BGA measurement using partial coherence sources," J. Opt. Soc. Korea 15, 352-356 (2011).

11. J. Huisken and D. Stainier, "Even fluorescence excitation by multidirectional selective plane illumination microscopy," Opt. Lett. 32, 2608-2610 (2007).

12. M. Dogar, H. Ilhan, and M. Ozcan, "Real-time, auto-focusing digital holographic microscope using graphics processors," Opt. Express 84, 704-716 (2013).

13. M. Baklanov, K. Maex, and M. Green, Dielectric Film for Advanced Microelectronics (Wiley-VCH, Berlin, 2007), Chapter 6.

14. T. lkeda, G. Popescu, and R. Dasari, "Hibert phase microscopy for investigating fast dynamics in transparent system," Opt. Lett. 30, 1165-1167 (2005).

15. W. Schleich, Quantum Optics in Phase Space (Wiley-VCH, Berlin, 2001), Chapter 2-5.

16. J. Rosen and G. Brooker, "Fluorescence incoherent color holography," Opt. Express 15, 2244-2250 (2007).

17. G. Phillips, "Green fluorescent protein - A bright idea for the study of bacterial protein localization," FEMS Microbio. Lett. 204, 9-18 (2001). 